\title{
Algorithm of Synthesizing on-Earth Scene Radar Images in Passive SAR Being the Part of Airborne bi-Static Radar System of General Configuration
}

\author{
S.G. Likhansky, S.B. Alekseev, T.A. Lepekhina, V.I. Nikolaev \\ Joint-Stock Corporation «Vega», Moscow, tatonika@inbox.ru
}

\begin{abstract}
The algorithm of radar image synthesis is proposed for general-configuration airborne bi-static look, in which the carriers (planes) of bi-static pair have the arbitrary different constant velocity vectors and fly on constant heights. The bi-static analog of Spotlight Mode is considered. Both the a-prior formation of secondary data arrays being necessary for image synthesis and the step-by-step illustration of synthesizing algorithm are being considered here.
\end{abstract}

Keywords - Fourier transform, Legendre transform, Taylor series, direct convolution, binary series, bi-static look, synthetic aperture radar, reference function, response Introduction

The bi-static SAR working modes provide some advantages, such as the following ones: high-quality image receiving along the flight direction, latent imaging, signal/active-shuffle ratio improvement and rising probability of detection objects made with the usage of «Stells» technology. These advantages of bi-static SAR are being caused by specific look geometry, but just this fact makes the processes of synthesizing radar aperture and of hologram processing more complicated.

The paper is devoted to actual problem of synthesizing the single look complex (SLC) radar images, signed in this paper as CRI, in airborne bi-static look. The aircraft are flying uniformly and straightly with arbitrary angle between their trajectories. Also, the case when one of the carriers is still on-earth station discussed here.

The analog of Spotlight Mode is being discussed. The active SAR-1 lights a-prior chosen still on-earth scene, the diagram of emitting (DE) of the passive SAR-2 is directed into SAR-1 vision point [1] (scene center) during the flight. Suppose the both radars are being able to realize electronic ray steering and flexible forming DE (for example - the Active Phased Antenna Array).

The hologram is formed in passive SAR-2. The following topics are discussed in this paper in detail:

1) A-prior formation of information arrays for synthesis using the primary navigation data;

2) All stages of image synthesizing algorithm. (Suppose the hologram is compressed by range.)

Such topics as A-posterior synthesis time interval, optimal choice of repetition frequency, coherent accumulation of DRH [5] are not discussed in this paper.

\section{THE PRIMARY NAVIGATION AND SAR DATA}

\section{A Bi-static SAR look geometry}

Suppose both the carriers of active SAR-1 and of passive SAR-2 flying uniformly and straightly on constant heights with arbitrary angle between motion directions.

All the processes are flowing in euclydian coordinate system (CS) $\bar{y} \equiv\left(y_{1}, y_{2}, y_{3}\right)$ having null-offset in the center of scene, being en-lighted by active SAR-1 ray.

Let the index numbers of scene and scene image are being signed as $\mathrm{X}, \mathrm{Y}$. Then the equality is correct, where $s$ - the step value between scene array indexes chosen with the restriction, where $\delta_{\text {min }}-$ minimal resolution interval for current look geometry.

The main values indications are being the follows:

$f$ - center carrier frequency; - band-width;

$F_{q} \approx(1,2 \ldots 1,3) \cdot B$ - quantization frequency;

$\bar{X}^{(k)}=\left(X_{1}^{(k)}, X_{2}^{(k)}, X_{3}^{(k)}\right), k=1,2$,

- SAR-1, SAR-2 - places in the moment $t=0$ in scene-CS;

$$
\bar{V}^{(k)}=\left(V_{1}^{(k)}, V_{2}^{(k)}, V_{3}^{(k)}\right)=\text { const }, k=1,2 ; V_{3}^{(k)}=0
$$

- SAR-1 and SAR-2 velocity vectors in scene-CS;

Vector absolute values: $X^{(1)}, X^{(2)} ; V^{(1)}, V^{(2)}$;

$F_{r}$ - SAR-1 pulses repetition frequency;

$H(x, r)$ - digital radio-hologram (DRH), being formed in passive SAR-2 - complex array having two indexes: $x$ (along-way) и $r$ (by-range);

$F(p, q)-$ DRH spectrum (shifted 2-dimention FFT of DRH) - complex array having two indexes $p$ (along-way) and $q$ (by-range) in spectral domain;

$N_{x} \times N_{r}-$ size of DRH and DRH spectrum arrays;

$K \times K-$ size of scene array (step value ). 
II. A-PRIOR ESTIMATION OF MAXIMUM GEOMETRIC RESOLUTION AND OF APPROPRIATE SYNTHESIS INTERVAL

\section{A Maximum resolution estimation}

The components $\mathbf{r}_{1}, \mathbf{r}_{2}$ of range SAR-1 - target SAR-2 $\}$ gradient $\nabla R(\bar{y})$ by $y_{1}, y_{2}$ and its module in scene center are being the following, correspondingly:

$$
\begin{gathered}
\nabla R(\overline{0}) \equiv\left(r_{1}, r_{2}\right) \equiv\left(X_{1}^{(1)} / X_{1}^{(1)}, X_{1}^{(2)} / X_{1}^{(2)}\right) \cdot f / c, \\
a_{R} \equiv|\nabla R(\overline{0})| \equiv\left(r_{1}^{2}+r_{2}^{2}\right)^{1 / 2} .
\end{gathered}
$$

The interval of maximum resolution $\delta_{\text {min }}(\mathrm{m})$ :

$$
\delta_{\min }=c / B \cdot 1 / a_{R} \text {. }
$$

B Estimation of synthesis time interval providing maximum resolution

The gradient by $y_{1}, y_{2}$ of Doppler shift $\delta f(\bar{y})$ along the line SAR-1 - target - SAR-2 $\}$ and its components:

$$
\begin{aligned}
& \nabla \delta f(\overline{0}) \equiv\left(f_{1}, f_{2}\right) \equiv\left(\partial(\delta f(\overline{0})) / \partial y_{1}, \partial(\delta f(\overline{0})) / \partial y_{2}\right), \\
& f_{k}=\frac{f}{c} \cdot \sum_{n=1}^{2}\left(V_{k}^{(n)} / X^{(n)}-X_{k}^{(n)} \cdot\left(\bar{X}^{(n)}, \bar{V}^{(n)}\right) /\left(X^{(n)}\right)^{3}\right) .
\end{aligned}
$$

The absolute value of gradient (4) equal $\mathrm{a}_{\mathrm{f}}=\left(\mathrm{f}_{1}^{2}+\mathrm{f}_{2}^{2}\right)^{1 / 2}\left(\mathrm{~m}^{-1} \mathrm{~s}^{-1}\right)$, synthesis interval $\boldsymbol{T}$ (s), providing maximum resolution (3) in scene center, equal:

$$
T \approx(1.3 \ldots 1,7) \cdot B / c \cdot \mathrm{a}_{\mathrm{R}} / \mathrm{a}_{\mathrm{f}} \cdot 1 /|\sin \vartheta|
$$

where $\sin \vartheta:=\left(\mathrm{r}_{1} \cdot \mathrm{f}_{2}-\mathrm{r}_{2} \cdot \mathrm{f}_{1}\right) /\left(\mathrm{a}_{\mathrm{R}} \cdot \mathrm{a}_{\mathrm{f}}\right)$ - sinus of the angle between two gradients (1) и (4).

\section{A-PRIOR CALCULATION DATA FOR SYNTHESIZING USING PRIMARY NAVIGATION DATA}

The synthesizing CRI data in current look is following:

1) Two-parameter X, Y - aggregate (family, set) of 2dimention spectral references for synthesizing;

2) Inverse geocoding law, mapping each scene point $X$, $\mathrm{Y}$ into the pair of DRH-indexes $x, r$, responding to he stable point of range migration law.

\section{A Introductory comments}

The term «current variable scene point» $\bar{y}$ in chapter IV (if the inverse is not adopted) means one of following 6 points: 1) scene center , 2) «very-close» point of x-axis , 3) «very-close» point of y-axis , 4) «not very close» point of $\mathrm{x}$-axis , 5) «not very close» point of $\mathrm{y}$-axis
$\left.\Delta_{2} \bar{y} \equiv\left(\begin{array}{lll}0 & \mathrm{E} & 0\end{array}\right), 6\right)$ «not very close» point $\Delta_{12} \bar{y} \equiv\left(\begin{array}{lll}\mathrm{E} & \mathrm{E} & 0\end{array}\right) \quad$ on diagonal line. ( $\varepsilon \approx 0,005 \ldots 0,010(m), \mathrm{E} \approx 1 \ldots 2(m)$.)

These six of points is being introduced with the purpose to estimate the coefficients of variations of migration law Legendre Transform [2, 3, 4] (see later) when point $\bar{y}$ varies within scene. These coefficients are being calculated up to 2-degree of Taylor series from scene coordinates $\bar{y}$.

$B$ Algorithm of migration law expanding into by-time Taylor series using FFT

Consider two formulae: The slant range (along the line \{SAR-1 - on-earth point - SAR-2\}) migration law, $R(\bar{y} ; t), t \in[-T / 2, T / 2], T$ is defined in (6):

$$
R(\bar{y} ; t)=\sum_{k=1}^{2}\left(\left|\bar{X}^{(k)}-\bar{y}\right|^{2}+\left(V^{(k)}\right)^{2} \cdot t^{2}\right)
$$

The law (7) after compensation of linear part of scene center migration law (let's call it «reduced migration law»):

$$
R(\bar{y} ; t)=R(\bar{y} ; t)-\left(\sum\left(\bar{X}^{(k)}, \bar{V}^{(k)}\right) / X^{(k)}\right) \cdot t
$$

Just the reduced law (8) appears in synthesis algorithm.

The FFT-algorithm of (8) expansion by time, discussed then, is being fulfilled in each of above mentioned 6 points.

Define the FFT base the following: $\mathrm{N} \approx 2^{12} \ldots 2^{13}$.

Let's calculate the values of formula (8) left part in the following $\mathrm{N}$ complex «time points»:

$$
\begin{gathered}
t_{n} \equiv 0,5 T \omega_{N}^{n} \equiv 0,5 T \exp (j 2 \pi \cdot n / N), n=0,1, \ldots, N-1 \\
Z(\bar{y} ; n):=R\left(\bar{y} ; 0,5 T \omega_{N}^{n}\right) .
\end{gathered}
$$

The processing of N-size array (9) with non-shifted FFT on base $\mathrm{N}$ provides the output complex array $W(\bar{y} ; n), n=0,1, \ldots, \mathrm{N}-1$. The correction by norm of the FFT-output array by the usage of formula

$$
w(\bar{y} ; n):=W(\bar{y} ; n) / \sqrt{N} \cdot(2 / T)^{n},
$$

provides the array (10) of Taylor coefficients of «centercompensated» migration law (8):

$$
\mathrm{R}(\bar{y} ; t) \approx \sum_{n=1}^{N-1} w(\bar{y} ; n) \cdot t^{n}
$$

In expansion (11) the degree $\mathrm{N}$ is being decreased to $N \approx 2^{6} \ldots 2^{7}$, just the restricted expansion (11) provides high-exactness $\sim(\mathrm{m})$ approximation of (8). 
C Legendre Transform of migration law with the move to radial-velocity domain

Taylor series of reduced radial velocity

The Taylor series coefficients for 1-derivative of law

(8) by time are being the follows:

$$
w^{(1)}(\bar{y} ; n-1) \equiv n \cdot w(\bar{y} ; n), 1 \leq n \leq N-1 .
$$

The Taylor series with the coefficients (12) is convergent to center-compensated (i.e. reduced) radial velocity:

$$
v_{\text {rad }}(\bar{y} ; t) \approx \sum w^{(1)}(\bar{y} ; n) \cdot t^{n} .
$$

Algorithm of Taylor expansion time by reduced radial velocity using FFT-method

Let's expand into Taylor series the function of time being inverse to (13) - the function of depending time from reduced radial velocity: . Let's resolve the following equation relatively unknown variable $t$ :

$$
v_{\text {rad }}=w^{(1)}(\bar{y} ; 0)+w^{(1)}(\bar{y} ; 1) \cdot t+\sum w^{(1)}(\bar{y} ; n) \cdot t^{n},
$$

for complex «velocity points»: , where, and constant V has the value:

$$
V \equiv \min _{i=0,1}\left\{\sum r_{n}^{(1)}(\overline{0}) \cdot(-1)^{n \cdot i} \cdot T^{n} / 2^{n} \mid\right\} .
$$

Let's start the iteration procedure with the counter $i$.

Null iteration $i:=0 \quad-$ M-size complex array ( $m=0,1, \ldots, M-1$ ), defining by the formula:

$$
t_{m}^{(0)}(\bar{y}):=0,5 \cdot V \cdot \omega_{M}^{m} / w^{(1)}(\bar{y} ; 1)
$$

Each procedure step re-calculates M-size complex array according the following formula:

$$
\begin{aligned}
& t_{m}^{(i+1)}(\bar{y}):=t_{1}^{(i)}(\bar{y})+\left(0,5 \cdot V \cdot \omega_{M}^{m}-\right. \\
& \left.-\sum w^{(1)}(\bar{y} ; n) \cdot\left(t_{m}^{(i)}(\bar{y})\right)^{n}\right) / w^{(1)}(\bar{y} ; 1) .
\end{aligned}
$$

The condition of exit from iteration procedure (17): $\left.\max _{m} \mid t_{m}^{\left(i_{\text {end }}+1\right)}(\bar{y})-t_{m}^{\left(i_{\text {end }}\right.}\right)(\bar{y}) \mid<\left(10^{-13} \ldots 10^{-14}\right)$,

The received array $t_{m}^{\left(i_{\text {end }}\right)}(\bar{y}), m=0,1, \ldots, \mathrm{M}-1$ is being indicated $z(\bar{y} ; m), m=0,1, \ldots, M-1$. The processing of this array with non-shifted FFT on base $\mathrm{M}$ provides the output array $w(\bar{y} ; m), m=0,1, \ldots, M-1$.

After the following correction output array by norm

$$
u(\bar{y} ; m):=w(\bar{y} ; m) / \sqrt{M} \cdot(2 / V)^{m},
$$

$m=0,1, \ldots, M-1$, we receive Taylor coefficients, providing the dependence time from reduced velocity :

$$
t=t\left(\bar{y} ; v_{\text {rad }}\right) \approx \sum_{m=0}^{M-1} u(\bar{y} ; \quad m) \cdot v_{\text {rad }}^{m}
$$

the expansion is being restricted: .

Taylor expansion of Legendre Transform and its variations by reduced radial velocity

The Legendre Transform [2, 3, 7, 8] of range migration law (8) is being represented by the formula:

$$
L\left(\bar{y} ; v_{\text {rad }}\right) \equiv t\left(\bar{y} ; v_{\text {rad }}\right) \cdot v_{\text {rad }}-R\left(\bar{y} ; t\left(\bar{y} ; v_{\text {rad }}\right)\right) \text {, }
$$

where is sum of series (19).

Let's expand $L\left(\bar{y} ; \quad v_{\text {rad }}\right)$ into Taylor $v_{\text {rad }}$-series. For solving the task, calculate the function $L\left(\bar{y} ; \quad v_{\text {rad }}\right)$ value in $\mathrm{L} \approx 2^{10} \ldots 2^{11}$ complex «radial-velocity points» $v_{\text {rad, } l}:=0,5 \mathrm{~V} \omega_{\mathrm{L}}^{l} \equiv 0,5 \mathrm{~V} \exp (j 2 \pi \cdot l / \mathrm{L}), l=0,1, \ldots, \mathrm{L}-1$ :

$$
G(\bar{y} ; l):=L\left(\bar{y} ; 0,5 V \omega_{L}^{l}\right)
$$

by usage of sum (19) value (expansion ) substitution into formula (20) and of Taylor t-expansion (11) of migration law (8). The processing of L-size complex array (21) $G(\bar{y} ; l)$ with non-shifted FFT on base L provides complex array $H(\bar{y} ; \quad l), l=0,1, \ldots, \mathrm{L}-1$. The correction this array by norm by usage of the formula

$$
h(\bar{y} ; l):=H(\bar{y} ; l) / \sqrt{\mathrm{L}} \cdot(2 / \mathrm{L})^{l},
$$

provides the array (22) is being the wanted array of Taylor coefficients of Legendre Transform:

$$
L\left(\bar{y} ; v_{\text {rad }}\right) \approx \sum h(\bar{y} ; m) \cdot v_{\text {rad }}^{l}
$$

degrees are below-restricted $l \geq 2$ for response correct mobility - according to the Inverse geocoding law.

The expansion (23) is being received for described in introduction part 6 special points. Let's apply these for calculating by-scene variations of Taylor coefficients (23) of Legendre Transform (formula (24) below):

$$
\begin{aligned}
& L\left(\bar{y}(\mathrm{X}, Y) ; v_{\text {rad }}\right) \approx \sum\left(c_{0}^{(l)}+c_{1}^{(l)} \cdot X+c_{2}^{(l)} \cdot Y\right) \cdot v_{\text {rad }}^{l}+ \\
& +\sum\left(c_{11}^{(l)} \cdot X^{2}+c_{12}^{(l)} \cdot \mathrm{XY}+c_{12}^{(l)} \cdot Y^{2}\right) \cdot v_{\text {rad }}^{l} .
\end{aligned}
$$

The coefficients of X,Y-linear terms in (24) are being calculated by usage of the following formulae: 


$$
\begin{aligned}
& c_{0}^{(l)} \approx h(\overline{0} ; l) ; c_{1}^{(l)} \approx\left(h\left(\delta_{1} \bar{y} ; l\right)-h(\overline{0} ; l)\right) \cdot s / \varepsilon ; \\
& c_{2}^{(l)} \approx\left(h\left(\delta_{2} \bar{y} ; l\right)-h(\overline{0} ; l)\right) \cdot s / \varepsilon .
\end{aligned}
$$

via the arrays of formula (24) in 6 special points.

The coefficients of X,Y -quadric terms (24) are being calculated using (25):

$$
\begin{aligned}
& c_{k k}^{(l)} \approx\left(h\left(\Delta_{k} \bar{y} ; l\right) \cdot s^{2}-c_{k}^{(l)} \cdot \mathrm{E} \cdot s-h(\overline{0} ; l) \cdot s^{2}\right) / \mathrm{E}^{2}, \\
& c_{12}^{(l)} \approx\left(\begin{array}{l}
h\left(\Delta_{12} \bar{y} ; l\right) \cdot s^{2}-\left(c_{11}^{(l)}+c_{22}^{(l)}\right) \cdot \mathrm{E}^{2}- \\
-\left(c_{1}^{(l)}+c_{2}^{(l)}\right) \cdot \mathrm{E} \cdot s-h(\overline{0} ; l) \cdot s^{2}
\end{array}\right) / \mathrm{E}^{2},
\end{aligned}
$$

where $k=1,2$.

\section{Secondary navigation data - references and coeffi- cients of their phase functions}

Moving Legendre Transform of migration law from radial-velocity domain to domain of pair of spectral non-sized indexes (i.e. offset numbers)

Let's scale coefficients $(25,26)$ for moving from radial-velocity scale into non-sized scale of spectral offsets numbers (array indexes) $p, q$ and also for moving from range $N_{r}$ and along-way $N_{x}$ bases to scene array base $K$ [7]:

$$
\begin{aligned}
& a_{k}^{(l)}:=c^{l-1} \frac{K N_{r}^{l-1}}{N_{x}^{l}} \frac{F_{r}^{l}}{F_{q}^{l-1}} \cdot c_{k}^{(l)}, k=0,1,2 ; \\
& a_{i j}^{(l)}:=c^{l-1} \frac{K N_{r}^{l-1}}{N_{x}^{l}} \frac{F_{r}^{l}}{F_{q}^{l-1}} \cdot c_{i j}^{(l)}, 1 \leq i, j \leq 2 .
\end{aligned}
$$

for numbers $2 \leq 1 \leq L-1$ (c - light velocity); carrier frequency $f$ move into scale of non-sized offsets by range:

$$
Q:=f \cdot N_{r} / F_{q} .
$$

The coefficients $(25,26)$ appear (just in scale (27) [7, 8]) in phase functions of references for synthesizing CRI.

\section{Spectral references for sinthesis}

Two factors of the X, Y-set of references with linear and quadric phases by $\mathrm{X}, \mathrm{Y}$ are being such ones $[7,8]$ :

$$
\begin{aligned}
& \mathrm{G}_{1}(\mathrm{X}, \mathrm{Y} ; p, q) \approx \exp \left(j \frac{2 \pi}{K} \cdot \sum_{l=2}^{L-1}\left(\begin{array}{c}
a_{0}^{(l)}+ \\
a_{1}^{(l)} \mathrm{X}+a_{2}^{(l)} \mathrm{Y}
\end{array}\right) \frac{p^{l}}{(Q+q)^{l-1}}\right), \\
& \mathrm{G}_{2}(\mathrm{X}, \mathrm{Y} ; p, q) \approx \exp \left(j \frac{2 \pi}{K} \cdot \sum_{l=2}^{L-1}\left(\begin{array}{c}
a_{11}^{(l)} \mathrm{X}^{2}+a_{22}^{(l)} \mathrm{Y}^{2} \\
+a_{12}^{(l)} \mathrm{XY}
\end{array}\right) \frac{p^{l}}{(Q+q)^{l-1}}\right) .
\end{aligned}
$$

$G_{0}(p, q) \approx \exp \left(j \cdot 2 \pi / K \cdot \sum a_{0}^{(l)} \cdot p^{l} /(Q+q)^{l-1}\right)$.

\section{Preliminary step of synthesis algorithm}

Let's compensate the center phase factor in DRH spectrum $F(p, q)$ by its multiplying on center reference (31):

$$
f(p, q):=G_{0}(p, q) \cdot F(p, q) .
$$

The integral formula of synthesis moves to look such:

$$
\begin{aligned}
& \mathrm{I}(\mathrm{X}, \mathrm{Y}) \approx \iint d p d q f(p, q) \times \\
& \times \exp \left(j \cdot\left(2 \pi / N_{x} \cdot p \cdot x(\mathrm{X}, \mathrm{Y})+2 \pi / N_{r} \cdot q \cdot r(\mathrm{X}, \mathrm{Y})\right)\right) \times \\
& \times \exp \left(\frac{j 2 \pi}{K} \cdot \sum_{l=2}^{L-1}\left(\begin{array}{c}
a_{1}^{(l)} \mathrm{X}+a_{2}^{(l)} \mathrm{Y}+ \\
a_{11}^{(l)} \mathrm{X}^{2}+a_{22}^{(l)} \mathrm{Y}^{2} \\
+a_{12}^{(l)} \mathrm{XY}
\end{array}\right) \frac{p^{l}}{(Q+q)^{l-1}}\right) .
\end{aligned}
$$

\section{Inverse geocoding formulae}

The inverse geocoding functions (appearing above - in phase of Fourier-exponent in (33)) are mapping pointtarget coordinates $\mathrm{X}, \mathrm{Y}$ into the response indexes $x, r$ of CRI array if synthesizing in way-range coordinates:

$$
\begin{aligned}
& x(\mathrm{X}, \mathrm{Y}) \approx F_{r} \cdot \sum_{m=0}^{M-2} u(\bar{y}(\mathrm{X}, \mathrm{Y}) ; m) \cdot\left(v_{r a d}(\bar{y}(\mathrm{X}, \mathrm{Y}) ; 0)\right)^{m}, \\
& r(\mathrm{X}, \mathrm{Y}) \approx \frac{F_{q}}{c} \cdot \sum_{n=1}^{N-1} w(\bar{y}(\mathrm{X}, \mathrm{Y}) ; n)\left(x(\mathrm{X}, \mathrm{Y}) / F_{r}\right)^{n} .
\end{aligned}
$$

The radial velocity (when $t=0$ ) in (34) equal:

$$
v_{\text {rad }}(\bar{y}(\mathrm{X}, \mathrm{Y}) ; 0)=-\sum_{k=1}^{2} \frac{\left(\bar{X}^{(k)}-\bar{y}(\mathrm{X}, \mathrm{Y}), \bar{V}^{(1)}\right)}{\left|\bar{X}^{(k)}-\bar{y}(\mathrm{X}, \mathrm{Y})\right|}
$$

Supplying to functions (34) difference-approximation derivations of the orders 1 and 2 almost like in formulae $(25,26)$, we receive the 2-degree expansion:

$$
\left(\begin{array}{l}
x(\mathrm{X}, \mathrm{Y}) \\
r(\mathrm{X}, \mathrm{Y})
\end{array}\right) \approx\left(\begin{array}{ll}
\alpha & \beta \\
\gamma & \delta
\end{array}\right)\left(\begin{array}{l}
\mathrm{X} \\
\mathrm{Y}
\end{array}\right)+\left(\begin{array}{l}
\mathrm{X} \\
\mathrm{Y}
\end{array}\right)^{\mathrm{T}}\left(\begin{array}{ll}
\eta_{11} & \eta_{12} \\
\eta_{12} & \eta_{22}
\end{array}\right)\left(\begin{array}{l}
\mathrm{X} \\
\mathrm{Y}
\end{array}\right) .
$$

The coefficients of formula (36) are being used twice in the paper: on stage of Generalized Stolt Transform and on stage of precise final focusing of CRI.

The scene-center reference is being such one $[7,8]$ : 
IV. FIRST STEP OF CRI SYNTHESIZING ALGORITHM APPROXIMATE INTEGRAL SYNTHESIZING OPERATOR TRANSFORM INTO IFFT-OPERATOR

A Approximation of synthesizing operator having phase function of reference being linear by scene coordinates and accounting linear part of Inverse geocoding law

This approximation of synthesizing operator is being received from formula (33) via formula (36):

$$
\mathrm{I}_{1}(\mathrm{X}, \mathrm{Y}) \approx \iint d p d q \cdot f(p, q) \times
$$

$$
\begin{aligned}
& \times \exp \left(\frac { j 2 \pi } { K } \left(( Q + q ) \left(\begin{array}{c}
\gamma+\alpha \frac{p}{Q+q}+ \\
\left.\left.\left.+\sum_{l=2}^{L-1} a_{1}^{(l)}\left(\frac{p}{Q+q}\right)^{l}\right)-Q \gamma\right) \cdot \mathrm{X}\right) \times
\end{array}\right.\right.\right. \\
& \times \exp \left(\frac { j 2 \pi } { K } \left(( Q + q ) \left(\begin{array}{l}
\delta+\beta \frac{p}{Q+q}+ \\
\left.\left.\left.+\sum_{l=2}^{L-1} a_{2}^{(l)}\left(\frac{p}{Q+q}\right)^{l}\right)-Q \delta\right) \cdot \mathrm{Y}\right) .
\end{array}\right.\right.\right.
\end{aligned}
$$

The coefficients $\alpha, \beta, \gamma, \delta$ are being re-scaled coefficients $\alpha, \beta, \gamma, \delta$ from formula (36) - with the aim of moving from DRH-scale to CRI-scale:

$$
\begin{aligned}
& \alpha:=\alpha \cdot K / N_{x}, \beta:=\beta \cdot K / N_{r}, \\
& \gamma:=\gamma \cdot K / N_{x}, \delta:=\delta \cdot K / N_{r} .
\end{aligned}
$$

The substitution of spectral variables in (37), vitally improving the Stolt Transform [2, 3, 7, 8], is being exist:

$$
\begin{aligned}
& W:=(Q+q) \cdot\left(\gamma+\alpha \frac{p}{Q+q}+\sum_{l=2}^{L-1} a_{1}^{(l)}\left(\frac{p}{Q+q}\right)^{l}\right)-Q \gamma, \\
& Z:=(Q+q) \cdot\left(\delta+\beta \frac{p}{Q+q}+\sum_{l=2}^{L-1} a_{2}^{(l)}\left(\frac{p}{Q+q}\right)^{l}\right)-Q \delta .
\end{aligned}
$$

Let’s call (39) «Generalized Stolt Transform (GST)».

In these new spectral indexes $W, Z$ (39) operator of synthesis (37) is moving into IFFT on base $K \mathrm{x} K$ :

$$
I_{1}(\mathrm{X}, \mathrm{Y}) \approx \iint \begin{gathered}
d W d Z \exp (j \cdot 2 \pi / K \cdot(W \cdot X+Z \cdot Y)) \times \\
\times J(W, Z) \cdot f(W, Z)
\end{gathered}
$$

where $\mathrm{J}(W, Z) \equiv J(\mathrm{p}(W, Z), \mathrm{q}(W, Z))$ - Jacobian of substitution $\mathrm{p} \equiv \mathrm{p}(W, Z), \mathrm{q} \equiv \mathrm{q}(W, Z)$ being inverse

to

GST:

$$
J(W, Z) \equiv 1 /\left|\begin{array}{cc}
\alpha+\sum_{l=2}^{L-1} l a_{1}^{(l)}(p /(Q+q))^{l-1} & \gamma-\sum_{l=2}^{L-1}(l-1) a_{1}^{(l)}(p /(Q+q))^{l} \\
\beta+\sum_{l=2}^{L-1} l a_{2}^{(l)}(p /(Q+q))^{l-1} & \delta-\sum_{l=2}^{L-1}(l-1) a_{2}^{(l)}(p /(Q+q))^{l}
\end{array}\right|,
$$

and $\mathbf{f}(\boldsymbol{W}, Z)$ - spectrum (32) of center-compensated $\mathrm{DRH}$ in variables

$$
f(W, Z) \equiv f(p(W, Z), q(W, Z)) .
$$

\section{B Generalized Stolt Transform algorithmic realization} Preliminary regularization of GST-formulae

The variables transform (39) can be realized by sequence of one-dimension scalings and shifts $[1,6]$.

The following substitution of output variables $W, Z \rightarrow W, Z$ and coefficients in (39) is being useful:

$$
\left(\begin{array}{ll}
w & b_{1}^{(l)} \\
z & b_{2}^{(l)}
\end{array}\right):=|\alpha \delta-\beta \gamma|^{-1}\left(\begin{array}{cc}
\delta & -\gamma \\
-\beta & \alpha
\end{array}\right)\left(\begin{array}{cc}
W & a_{1}^{(l)} \\
Z & a_{2}^{(l)}
\end{array}\right) .
$$

The combination (41) of formulae (39) is moving GST to the form (42), simply decomposing into few number of one-dimension scalings and shifts (4 steps):

$$
\begin{aligned}
& w:=p \cdot\left(1+\sum_{l=2}^{L-1} b_{1}^{(l)} \cdot(p /(Q+q))^{l-1}\right), \\
& Q+z:=(Q+q) \cdot\left(1+\sum_{l=2}^{L-1} b_{2}^{(l)} \cdot(p /(Q+q))^{l}\right) .
\end{aligned}
$$

\section{Realization of the first (principal) GST stage}

Transform (42) $p, q \rightarrow w, z$ can be realized by 4 steps:

1) Scaling of «azimuth» $(p, q) \rightarrow(\varphi, q)$ :

$$
p \rightarrow \quad ; \quad:=p \cdot Q /(Q+q)
$$

2) Scaling-shift of «range» $(\varphi, q) \rightarrow(\varphi, z)$ :

$$
z:=\left(1+\sum_{l=2}^{L-1} b_{2}^{(l)}(\varphi / Q)^{l}\right) \cdot q+Q \cdot \sum_{l=2}^{L-1} b_{2}^{(l)}(\varphi / Q)^{l}
$$

3) Scaling of «azimuth» (non-linear one, but the same for each «range» offset) :

$$
\psi:=\varphi \cdot\left(1+\sum_{l=2}^{L-1} b_{1}^{(l)}(\varphi / Q)^{l-1}\right)\left(1+\sum_{l=2}^{L-1} b_{2}^{(l)}(\varphi / Q)^{l-1}\right)^{-1}
$$

4) Scaling of «azimuth» :

$$
\psi \rightarrow w ; \quad w:=(1+z / Q) \cdot \psi
$$

Realization of the second (final) stage of GST

This stage realizes the inverse linear substitution of variables $w, z \rightarrow W, Z$ with the matrix from (36).

Let's introduce the following values (using (36)): 


$$
\begin{aligned}
& \rho_{1}=\sqrt{\alpha^{2}+\gamma^{2}} ; \quad \rho_{2}=\sqrt{\beta^{2}+\delta^{2}} ; \\
& \varphi_{1}=\arg (\alpha+j \cdot \gamma) ; \varphi_{2}=\arg (\beta+j \cdot \delta) ; \psi \equiv \varphi_{1}-\varphi_{2} .
\end{aligned}
$$

The second stage can be decomposed into 5 steps (using the values (47)).

1) Scaling of «azimuth» :

$$
w \rightarrow w_{1} ; w_{1}:=\rho_{1} \cdot w ;
$$

2) Scaling of «range» :

$$
z \rightarrow z_{1} ; z_{1}:=\rho_{2} \sin \psi \cdot z ;
$$

3) Scaling-shift of «azimuth» $\left(w_{1}, z_{1}\right) \rightarrow\left(w_{2}, z_{1}\right)$;

$$
w_{2}:=\cos _{1} \cdot w_{1}+\cos { }_{1}\left(\cot \psi-\tan { }_{1}\right) \cdot z_{1}
$$

4) Scaling of «range» :

$$
Z_{1} \rightarrow Z ; \quad Z:=\sec \varphi_{1} \cdot z_{1}
$$

5) Scaling of «azimuth» :

$$
w_{2} \rightarrow W ; \quad W:=w_{2}+\tan \varphi_{1} \cdot Z .
$$

The terms «azimuth» and «range» are purely agreed.

Functional operator GST and its operands

The scalings (43-46, 48-52) are linear operators which act in linear space of complex arrays, re-presentable via FFT and multiplications with appropriate references $[1,6]$.

Scaling (43-46, 48-52) operands are being 2dimension complex arrays: $f(p, q) \cdot J(p, q)$ (multiplying of Jacobian of inverse GST transform and centercompensated spectrum (32)), and the arrays of index (offset) numbers: $p(p, q), q(p, q)$.

The scaled array $f(p, q) \cdot J(p, q)$ is being the following: $\mathrm{f}(W, Z) \cdot \mathrm{J}(W, Z)$, and it must be processed by IFFT (formula (40)) moving on output into CRI-1: $\mathrm{I}_{1}$ (X, $\mathrm{Y})$.

The arrays $\mathrm{p}(W, Z), \mathrm{q}(W, Z)$ are being used on steps 2, 3 of synthesis (chapter VI) - during CRI upfocusing.

V. SECOND AND THIRD STEPS OF CRI SYNTHESIZING ALGORITHM - IMAGE UP-FOCUSING USING REFERENCE FACTOR WITH QUADRIC PHASE BY SCENE COORDINATES

A Second step of algorithm - up-focusing using approximate expansion of reference having quadric phase by scene coordinates into binary series

The step consists of further focusing of primary image $\mathrm{I}_{1}(\mathrm{X}, \mathrm{Y})(\mathrm{CRI}-1)$ being the output of IFFT (40) after fin- ishing GST with usage the expansion into binary series the following «main sub-factor» of X, Y-quadric factor (30) of references whole X, Y-set:

$$
\begin{aligned}
& \mathrm{G}_{2}^{\text {Main }}(\mathrm{X}, \mathrm{Y} ; p, q) \approx \\
& \approx \exp \left(j \frac{2 \pi}{K}\left(\begin{array}{c}
a_{11}^{(2)} \mathrm{X}^{2}+a_{22}^{(2)} \mathrm{Y}^{2} \\
+a_{12}^{(2)} \mathrm{XY}
\end{array}\right) \cdot \sum_{l=2}^{L-1} d_{l} \frac{p^{l}}{(Q+q)^{l-1}}\right) .
\end{aligned}
$$

The view of reference (53) caused by idea of the best approximation of the vectors: $\bar{a}_{l} \equiv\left(a_{11}^{(l)}, a_{12}^{(l)}, a_{22}^{(l)}\right), l \geq 3$, composed of quadric form coefficients in phase (33), with the vector, collinear to . The coefficients $d_{l}, \quad l \geq 2$ in formula (53) are being such ones:

$$
d_{l}:=\left(\bar{a}_{2}, \bar{a}_{l}\right) /\left(\bar{a}_{2}, \bar{a}_{2}\right), l \geq 2 ; d_{2}=1 .
$$

The phase of reference (53) is binary - equal the multiplication of X, Y-pure function and W, Z-pure function.

The formula of synthesis (33) after GST (39) and extracting sub-factor (53) from reference moves to be such:

$$
\begin{aligned}
& \mathrm{I}(\mathrm{X}, \mathrm{Y}) \approx \iint d W d Z \exp (j \cdot 2 \pi / K \cdot(W \cdot \mathrm{X}+\mathrm{Z} \cdot \mathrm{Y})) \times \\
& \times \mathrm{J}(W, Z) \cdot \mathrm{f}(W, Z) \times \\
& \times \exp \left(j \frac{2 \pi}{K}\left(\begin{array}{r}
a_{11}^{(2)} \mathrm{X}^{2}+a_{22}^{(2)} \mathrm{Y}^{2} \\
+a_{12}^{(2)} \mathrm{XY}
\end{array}\right) \cdot \sum_{l=2}^{L-1} d_{l} \frac{p^{l}}{(Q+q)^{l-1}}\right) .
\end{aligned}
$$

The expansion of reference (53) into binary series in formula (54) of synthesis brings this formula to look as the integral-operator series with index $m=0 \ldots M$ :

$$
\begin{aligned}
& \mathrm{I}_{2}(X, Y) \approx \sum_{m=0}^{M}(j \cdot 2 \pi / K)^{m}\left(\sum_{i+k=2} a_{i k}^{(2)} X^{i} Y^{k}\right)^{m} / m ! x \\
& \iint^{M} d W d Z \exp (j \cdot 2 \pi / K \cdot(W \cdot X+Z \cdot Y)) x \\
& x J(W, Z) \cdot f(W, Z) \cdot\left(\sum_{l=2}^{L-1} d_{l} \cdot p^{l}(W, Z) /(Q+q(W, Z))^{l-1}\right)^{m} .
\end{aligned}
$$

The separation of variables into pure-coordinate and pure-spectral ones in each term (55) allows to process $W$, $Z$-factor of each term and so as to receive up-focused (additionally-focused) image $\mathrm{I}_{2}(\mathrm{X}, \mathrm{Y})(\mathrm{CRI}-2)$ by means of the $\mathrm{X}$, Y-coefficient linear combination of IFFT-processed and moved into $\mathrm{X}, \mathrm{Y}$-domain $\mathrm{W}, \mathrm{Z}$-factors (of each term).

$B$ Third (final) stage of algorithm - up-focusing by means of local direct convolution CRI-2 with residual references on short base

The residual X,Y-set of spectral references (after elimination sub-factor from factor $G_{2}$ ) look such: 


$$
\begin{aligned}
& G_{\text {Res }}(\mathrm{X}, \mathrm{Y} ; W, Z) \approx \\
& \exp \left(\begin{array}{l}
\left.j 2 \pi / K \cdot \sum_{l=2}^{L-1} \mathrm{p}^{l}(W, Z) /(Q+\mathrm{q}(W, Z))^{l-1} \cdot\right) \\
\sum_{m+n=2} \mathrm{X}^{m} \mathrm{Y}^{n}\left(a_{m n}^{(l)}-d_{l} \cdot a_{m n}^{(2)}\right)
\end{array}\right) \times \\
& \times \exp \left(j 2 \pi / K \cdot \sum_{m+n=2} \eta_{\mathrm{mn}} \cdot \mathrm{X}^{m} \mathrm{Y}^{n}\right),
\end{aligned}
$$

where the coefficients $\mathrm{m}_{k l}, \mathrm{n}_{k l}$ are appearing in 2-degrre term of Inverse geocoding law formula (36).

The spectrum of reference (56) has in time domain short ( $\sim K / 32$ indexes) carrier and depends from number $\mathrm{X}$, Y slowly. If the CRI-2 $I_{2}(X, Y)$ from formula (55) output is being divided into $\sim 32 \times 32$ quadrates, in frames of each of them can be used the same reference from X,Y-set (56).

So, with the purpose of final precise CRI up-focusing (CRI-2 $\rightarrow$ CRI) the following algorithm can be applied.

For each quadrate of divided CRI-2 $\mathrm{I}_{2}(\mathrm{X}, \mathrm{Y})$ we calculate (with 2-dimention shifted FFT) time-domain reference $\mathrm{g}_{\text {Res }}\left(\mathrm{X}_{i i}, \mathrm{Y}_{i j} ; \mathrm{X}, \mathrm{Y}\right)$, responding to current quadrate center: $-K / 64 \leq i i, j j \leq K / 64$.

For each quadrate $-K / 64 \leq i i, j j \leq K / 64$ we fulfill the direct convolution CRI-2 $\mathrm{I}_{2}(\mathrm{X}, \mathrm{Y})$, being calculated with the formula (55), with very short based $\sim K / 32 \mathrm{x}$ $K / 32$ time-domain reference $\mathrm{g}_{\text {Res }}\left(\mathrm{X}_{\mathrm{ij}}, \mathrm{Y}_{\mathrm{ij}} ; \mathrm{X}, \mathrm{Y}\right)$, counting the output values only within the current quadrate.

On the output of the third step - finally (precisely) focused array CRI I (X, Y).

\section{GLOBAL STRUCTURE OF CRI SYNTHESIZING ALORITHM}

\section{A General structure of a-prior processing}

1) A-prior estimation of maximum-possible resolution and of responding interval of synthesis;

2) Expansion of range migration law into by-time Taylor series using FFT-method;

3) Expansion of time from radial-velocity dependence function into by-velocity Taylor series using FFT-method;

4) Legendre Transform (of range migration law) and its by-coordinate variations up to second degree (within scene) calculation using FFT-method;

5) Inverse geocoding law calculation up to second degree by scene coordinates;

6) Calculation of coefficient arrays, defining the set of reference functions for synthesis - up to second degree by scene coordinates.

\section{B General structure of synthesizing procedure}

1) Two-dimension hologram spectrum calculation;

2) Center-scene phase law factor compensation in hologram spectrum;

3) Approximate reducing of synthesizing operator to IFFT-operator by usage of Generalized Stolt Transform (which is being fulfilled as operator acting in linear space of 2-dimension spectral data-arrays via the few number of 1-dimension scales and shifts);

4) Step-by-step focusing image focusing using IFFTprocessing of binary series, being the result of approximate expansion of reference multiplicand with quadric phase (by scene coordinates) into exponential series;

5) Up-focusing of image using local direct convolution on short base.

\section{CONCLUSION}

Theoretic exploration and computer modeling of synthesis in bi-static look of general type have demonstrated such fact - synthesis parameters (synthesizing interval, optimal repetition frequency and hologram array size) are very dependant from look geometry including movement law of carriers relatively the scene - not only from demanded resolution and band-width.

Also was showed, that the equivalent-very-large-squint look situation (with equivalent squint angle value up to $\sim 30^{\circ} \ldots 45^{\circ}$ ) is being typical in bi-statics. So, one must fulfill the a-prior compensation of scene-center linear migration and work with any look as if it is equivalent-side-lobe one. Since, in bi-static look the CRI initially is being synthesized in horizontal rectangle scene coordinates (and interval of synthesis is being chosen with appropriate length excess), this compensation don't bring the problem of distortion of coordinate-net and response-form.

The few amount of appearing in the paper numeric data (error estimations, step values, polynomial degrees, iteration numbers, recommended FFT-bases etc.) are being conclusions from some amount of computer experiments. 


\section{REFERENCES}

[1] Verba V.S., Neronskij L.B., Osipov I.G., Turuk V.E. Radiolokacionnye sistemy zemleobzora kosmicheskogo bazirovaniya (Space-borne Earth Surveillance Radar Systems). // Izdatel'stvo «Radiotekhnika», 2010.

[2] Mittermayer J., Moreira A., Loffeld O. High Precision Processing of Spotlight SAR Data Using the Extended Chirp Scaling Algorithm. // EUSAR'98, pp. 561-564, Friedrichshafen, Germany, May 1998.

[3] Ian G. Cumming, Frank H. Wong. Digital Processing of Synthetic Aperture Radar Data. Algorithms and Implementation. // ArtechHouse, Boston \& London, 1992.

[4] Carrara W.G., Goodman R.S., Majewski R.M. Spotlight Synthetic Aperture Radar. Signal Proc $\neg$ essing Algorithms // ArtechHouse, Boston\&London, 1995.

[5] Lihanskij S.G Modified sliding window method as the way of stroboscopic-effect compensation and simul-taneously improvement productivity of convolution synthesizing algorithms in Spotlight Mode.) // Naukoem $\neg$ kie tekhnologii, № 10, 2006.
[6] Lihanskij S.G. New technology of high-precision radar signal scaling as generalization of well-known Chirp-Scaling operator) // Naukoem $\neg$ kie tekhnologii, № 7-8, 2006, str. 2838.

[7] Lihanskij S.G. Modified algorithm Omega-KA for highprecision radar image synthesizing in Spotlight Mode in space-based SAR and it's ballistic support. The Proceedings of the International Conference devoted to scientific and technical problems of Earth Surveillance, patrol and control of complexes of pilotless flying apparates // Trudy mezhdunarodnoj konferencii po nauchno-tekhnicheskim problemam zemleobzora, dozora i upravleniya i kompleksov s bespilotnymi letatel'nymi apparatami, Moskva, OAO «Koncern «Vega»», $2013 \mathrm{~g}$.

[8] Lihanskij S.G. Modification of synthesizing radar image algorithm Omega-KA with the purpose of precision improvement // Nauchno-metodiche $\neg$ skij sbornik TSNIIMORF № 2 (532) ver' 2013. Inv. 55441/3. 